\title{
Impact of COVID-19 on Hepatocellular Carcinoma Management: A Multicountry and Region Study
}

\author{
Mihir Gandhi (iD) ${ }^{1-4}$ \\ Wen-Huan Ling (iD) ${ }^{5}$ \\ Chien-Hung Chen (iD) 6 \\ Joon Hyeok Lee ${ }^{7}$ \\ Masatoshi Kudo (iD ${ }^{8}$ \\ Rawisak Chanwat ${ }^{9}$ \\ Simone I Strasser (ID ${ }^{10}$ \\ Zhu Xu' \\ Soh-Han Lai ${ }^{5}$ \\ Pierce Kah-Hoe Chow (D) 12,13
}

'Biostatistics, Singapore Clinical Research Institute, Singapore; ${ }^{2}$ Centre for Quantitative Medicine, Duke-NUS Medical School, Singapore; ${ }^{3}$ Lien Centre for Palliative Care, Duke-NUS Medical School, Singapore;

${ }^{4}$ Tampere Center for Child, Adolescent, and Maternal Health Research, Tampere University, Tampere, Finland; ${ }^{5}$ Programme in Translational and Clinical Liver Research, National Cancer Centre Singapore, Singapore; ${ }^{6}$ Department of Internal Medicine, National Taiwan University Hospital, Douliu City, Yunlin, Taiwan; ${ }^{7}$ Division of Gastroenterology, Sungkyunkwan University School of Medicine, Samsung Medical Centre, Seoul, Korea; ${ }^{8}$ Department of

Gastroenterology and Hepatology, Kindai

University Hospital, Osaka, Japan; 'Department of Surgery, National Cancer Institute, Bangkok, Thailand; ${ }^{10} \mathrm{AW}$ Morrow Gastroenterology and Liver Centre, Royal Prince Alfred Hospital, Sydney, NSW, Australia; "Department of Interventional Therapy, Key Laboratory of Carcinogenesis and Translational Research (Ministry of Education/Beijing), Peking University Cancer Hospital and Institute, Beijing, People's Republic of China;

${ }^{12}$ Department of Hepatopancreatobiliary and

Transplant Surgery, National Cancer Centre Singapore and Singapore General Hospital,

Singapore; ${ }^{13}$ Surgery Academic Clinical Programme, Duke-NUS Medical School, Singapore

Correspondence: Pierce Kah-Hoe Chow Division of Surgery and Surgical

Oncology, National Cancer Centre

Singapore, II Hospital Crescent, 169610, Singapore

Tel +65 6576215 I

Email pierce.chow.k.h@singhealth.com.sg
Purpose: The COVID-19 pandemic has altered healthcare priorities which may adversely impact cancer management. We aimed to evaluate the impact of the pandemic on the diagnosis, treatment, and consultation methods for patients with hepatocellular carcinoma (HCC).

Patients and Methods: We conducted a survey among 27 hospitals from 14 Asia-Pacific countries, collecting hospital-level information on the number of newly diagnosed HCC cases during a pre-pandemic period (February to May 2019) and for the same period during the pandemic (February to May 2020). Information was also collected on delays in diagnosis and treatment, changes in treatment modalities and complication rates, changes in patient enrollment in clinical trials, and modes of patient consultation. The information was stratified by the Barcelona Clinic Liver Cancer (BCLC) stage.

Results: The survey included cohorts of 2789 and 2045 patients newly diagnosed with HCC during the pre- and pandemic period, respectively. A decline of $26.7 \%$ in new HCC cases was reported during the pandemic compared to the pre-pandemic. A sizable proportion of institutions reported delays in diagnosis $(48.2 \%$ in BCLC $0 / \mathrm{A} / \mathrm{B}$ and $51.9 \%$ in BCLC C), delays in treatment $(66.7 \%$ in $\mathrm{BCLC} 0 / \mathrm{A} / \mathrm{B}$ and $63.0 \%$ in $\mathrm{BCLC} \mathrm{C})$, changes in treatment modality (33.3\% in BCLC 0/A/B and $18.5 \%$ in BCLC C), an increase in treatment complications (about $15 \%$ across all BCLC stages), and no growth in clinical trial enrollments during the pandemic. Furthermore, there was a decline of $27.3 \%$ in face-to-face patient consultations and an increase of $18.3 \%$ in video/telephonic consultations during the pandemic. A considerable variation in changes in HCC management was observed among countries.

Conclusion: The COVID-19 pandemic has significantly impacted the management of HCC among Asia-Pacific countries. The impact varies according to the disease stage and country. Well thought-through long-term strategies are required to ameliorate the negative impact of the pandemic on HCC patients.

Keywords: hepatocellular carcinoma, liver cancer, COVID-19, Asia-Pacific

\section{Introduction}

Hepatocellular carcinoma (HCC) is the 5th most common cancer globally, and the 3rd most common cause of cancer deaths, afflicting approximately 900,000 people yearly. There is great geographical variation in the distribution of HCC, and approximately $80 \%$ of patients are found in Asia. ${ }^{1}$

In 2020, a global pandemic emerged due to the novel severe acute respiratory syndrome coronavirus 2 (SARS-CoV-2) ${ }^{2}$ which is now termed COVID-19 (COrona, VIrus Disease 2019). Patients with cancer are particularly vulnerable due to their acquired immunodeficiency from cancer or its treatment. Cancer patients above the age of 70 have a higher risk of severe infection if infected 
with COVID-19 than the rest of the population. ${ }^{3}$ Healthcare institutions worldwide have shifted their focus and re-directed their efforts towards confronting the major challenges the pandemic has imposed, and the management of patients with cancer has thus become a lesser priority. Several countries had temporarily suspended screening programs for various types of cancers, ${ }^{4}$ leading to inevitable delays in diagnosis, which reduces the chances for the early detection of cancer and the prompt initiation of treatment. Treatment pathways in cancer are also interrupted as COVID-19 created logistical difficulties due to restrictions and precautions. Finally, cancer patient enrolment into clinical trials was curtailed during COVID19, which imposed high costs in passthrough costs and patient outcomes. 5

Different countries adopted different restrictions and precautionary steps for their healthcare systems, and these very often also depended on the country's socioeconomic status. Regarding $\mathrm{HCC}$, there was a severe paucity of data on how the pandemic has affected diagnosis, treatment, and patient consultations for patients with HCC. Reports in this regard have been mainly on other types of cancer or specific to a particular hospital. Therefore, it is difficult to understand the overall impact of the pandemic at a regional and global level. ${ }^{4,6-8}$ A recent systematic review $^{9}$ that identifies studies evaluating the impact of COVID-19 on HCC found only one study ${ }^{10}$ from an Italian center with $42 \mathrm{HCC}$ patients. Furthermore, an international survey assessing the impact of the COVID-19 on liver cancer management also included just one center from Asia (Japan). ${ }^{11}$ Since most HCC cases are in AsiaPacific, a multicountry study from the Asia-Pacific region will be needed to understand the overall impact of the pandemic and inter-country differences. This study was conducted during the initial phase of the pandemic and adopted a multicountry approach. The primary objective was to estimate the impact of the pandemic in terms of reduction in the number of patients diagnosed with $\mathrm{HCC}$ during the pandemic compared to its pre-pandemic period. Secondary objectives included estimation of the percentage of healthcare institutions that experienced changes in the HCC treatment modality, delays in HCC treatment initiation, increases in the treatment complication rate, reductions in clinical trial enrollment rate, and changes in mode-of-patient consultation during the pandemic compared to pre-pandemic for the corresponding period in the Asia-Pacific.
During the early phase of the pandemic, there were some expectations that the pandemic would be swiftly brought under control, and many of the strategies adopted then reflected this expectation. Many countries have since experienced multiple waves of COVID-19 infection, and there is increasing recognition that long-term sustainable strategies are required. Our study's findings will help physicians optimize care pathways to enhance treatment outcomes for HCC patients for the remaining part of the pandemic. Policymakers will also benefit from data that quantifies the impact of the pandemic on HCC so that better public health policies can be developed to cope with this ongoing pandemic.

\section{Patient and Methods Study Design and Data Collection}

The study was an online survey among tertiary healthcare institutions from the Asia-Pacific Hepatocellular Carcinoma (AHCC) Trials Group, participating in the HCC Registry in Asia study (ClinicalTrials.gov Identifier: NCT03233360). The AHCC Trials Group is a collaborative research group involving more than 50 healthcare institutions from 17 countries with a common goal of seeking efficacious treatments for HCC and engaged in research for patients with HCC. The online survey was designed with REDCap (Research Electronic Data Capture) electronic data capture tools hosted at Singapore Clinical Research Institute (SCRI). ${ }^{12-14}$ A unique link was created for each institution which was sent to the institution representative who had access to their institution's patient data, specifically for diagnosis, treatment, and management related to HCC. The survey form was designed with inputs from the expert Steering Committee of the Registry and comprised eight multidisciplinary experts (surgical oncologist, medical oncologist, radiation oncologist, interventional radiologist, hepatologist, and medical statistician) from 7 Asia-Pacific countries with extensive research experience in HCC.

The survey asked to provide information at the institution-level on the number of patients diagnosed with HCC stratified by Barcelona Clinic Liver Cancer (BCLC) stages $^{15}$ during a pre-COVID-19 pandemic period (1st February 2019 to 31st May 2019) and for the same period with COVID-19 pandemic (that is, 1st February 2020 to 31 st May 2020). The pandemic time period for the survey was selected considering the majority of Asia-Pacific countries have observed at least one major wave of the 
pandemic and implemented some COVID-19 control measures before February 2020 (see Table 1). The survey also asked for information on the impact of the pandemic on delay in the diagnosis of HCC, delay in initiation of $\mathrm{HCC}$ treatment, selection of modality of $\mathrm{HCC}$ treatment, increase in complication rates with HCC treatment, and change in patient enrollment for clinical studies. The impact of the pandemic on the delay in the diagnosis of HCC was defined as the postponement causing at least a "somewhat" delay compared to the pre-pandemic period norms in the respective institutions. Similarly, the other outcomes were defined in the same way. This information collected was also stratified by the BCLC stage. Finally, the distribution of mode of patient consultations (face-toface in clinic, face-to-face in patient's home, remote consultation using video/telephone) during pre-and during the pandemic was collected. The survey form was sent to all 50 institution representatives, and three reminders were sent to complete the survey.

As this study did not include any individual patientspecific information, other than the aggregate data at the institution level, patient consent and ethics approval were not required.

\section{Statistical Methods}

The number of patients diagnosed with HCC was compared between a corresponding pre-pandemic period and during the pandemic. We anticipated a mild-to-moderate effect of the pandemic on the number of patients diagnosed with HCC. An estimated sample size of 27 institutions was required to estimate the pandemic impact with a precision \pm 0.33 effect size (mean change in the number of diagnosed patients divide by its standard deviation) ${ }^{16}$ with a $90 \%$ probability.

The number of patients diagnosed with HCC per institution during the pre-pandemic and pandemic periods was summarized using mean and standard deviation. The mean change in the number of patients per institution from the prepandemic period was estimated using the effect size (as defined above) along with its $90 \%$ two-sided confidence interval. In addition, the percentage change in the number of patients diagnosed with HCC per country was estimated as the difference in the number of patients diagnosed by all the participating institutions in the country during the pandemic and pre-pandemic divided by the number of patients diagnosed during the pre-pandemic period, multiplied by 100. The percentage changes in the diagnosis at the country level were also estimated by the BCLC stage. The percentage of institutions that reported delay in the diagnosis of HCC, delay in initiation of HCC treatment, change in complication HCC treatment complication, and change in HCC patients participating in clinical studies were summarized by the number of institutions and the percentage of total institutions that participated in the study. Finally, the mean change in percentage of patients with different mode-ofconsultations (face-to-face or video/telephonic) per institution was estimated as the mean difference in percentages per institution between the pandemic and pre-pandemic periods. A country-level change was estimated as the mean change among all institutions within the country.

\section{Results}

A total of 27 institutions from 14 countries responded to the survey. These institutions collectively represented a cohort of 2789 and 2045 patients newly diagnosed with HCC during the pre- and pandemic period of 1st February to 31st May in 2019 and 2020, respectively. The list of participating institutions is provided in Supplemental Materials (Appendix 1). Among the 14 countries that responded, all of them except one implemented social distancing, seven implemented state/country lockdowns, and ten implemented border control measures during the survey period (February to May 2020) (Table 1).

During the pre-pandemic period (February to May 2019), there were on average 103.2 (SD 193.0) new patients diagnosed with HCC per institution, which were reduced to 75.7 (SD 126.3) patients during the same period under study. This is a decline of average 27.6 patients per institution or $26.7 \%$ from the pre-pandemic period (effect size $=0.37 ; 90 \%$ confidence interval 0.04 to 0.697 ).

From the country-level perspective, 12 of 14 countries reported a decline in new cases of HCC diagnosed during the pandemic than its previous year (Figure 1), with the highest drop reported by Myanmar (65\%), followed by the Philippines (50\%), Indonesia (50\%), and Hong Kong $(44 \%)$. Only one country - Malaysia - reported an increase in the newly diagnosed patients, whereas there was no change in Brunei. Among newly diagnosed $\mathrm{HCC}$ patients, there was a decline of an average of $12.0 \%$, $22.6 \%$, and $23.1 \%$ of patients among BCLC $0 / \mathrm{A}, \mathrm{B}$, and $\mathrm{C}$, respectively, across the countries during the pandemic compared to the pre-pandemic period (Table 2).

A sizable proportion of institutions reported delays in diagnosis of new $\mathrm{HCC}$ cases $(48.2 \%$ for BCLC $0 / \mathrm{A} / \mathrm{B}$ and $51.9 \%$ for BCLC C), delays in initiating HCC treatment (66.7\% for BCLC 0/A/B and $63.0 \%$ for BCLC C), changes 
Table I Types of COVID-19 Measures and Controls Implemented by the Individual Country During the Period of February to May 2020

\begin{tabular}{|c|c|c|c|c|}
\hline Country & $\begin{array}{l}\text { Border Controls Measures } \\
\text { Implemented }{ }^{24}\end{array}$ & $\begin{array}{l}\text { State/Country Lockdowns } \\
\text { Implemented }^{25}\end{array}$ & $\begin{array}{l}\text { Social Distancing Policies } \\
\text { Implemented }\end{array}$ & $\begin{array}{l}\text { COVID-19 } \\
\text { Cases }^{\mathrm{a}, 27}\end{array}$ \\
\hline Australia & Yes & Yes & Yes & 7202 \\
\hline Brunei & Yes & Yes & Yes & $14 \mid$ \\
\hline China & Yes & Yes & Yes & $84, \mid 46$ \\
\hline Hong Kong & Yes & No & Yes & 1085 \\
\hline Indonesia & No & No & Yes & 26,473 \\
\hline Japan & Yes & No & Yes & $|6,75|$ \\
\hline Malaysia & Yes & Yes & Yes & 7819 \\
\hline Mongolia & Yes & No & Yes & 179 \\
\hline Myanmar & No & No & No & 224 \\
\hline Philippines & No & Yes & Yes & 18,086 \\
\hline Singapore & Yes & Yes & Yes & 34,884 \\
\hline South Korea & No & No & Yes & 11,503 \\
\hline Taiwan & Yes & No & Yes & 442 \\
\hline Thailand & Yes & Yes & Yes & 3081 \\
\hline
\end{tabular}

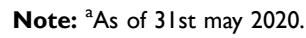

in treatment modality (33.3\% for BCLC 0/A/B and $18.5 \%$ for BCLC C), an increase in HCC treatment complications (14.8\% across all stages of BCLC), and no growth in clinical trial enrollments $(88.9 \%$ for BCLC 0/A, 81.5\% for BCLC B, and $70.4 \%$ for BCLC C) during the pandemic compared to the pre-pandemic period.

Among patients with the BCLC A stage, 51.9\% of institutions reported a reduction in the proportion of patients undergoing elective surgery (surgical resection or liver transplant) during the pandemic compared to the prepandemic period. Similarly, among patients with the BCLC B stage, $44.4 \%$ of institutions reported a reduction in the proportion of patients undergoing elective procedures (trans-arterial chemoembolization, surgery). However, $40.7 \%$ of institutions reported an increase in the proportion of patients treated with oral systemic HCC therapy (eg, Sorafenib, Lenvatinib) during the pandemic compared to the pre-pandemic period.

During the pandemic, there was a decline of $27.3 \%$ in face-to-face patient consultations and an increase of $18.3 \%$ in video/telephonic consultations per institution compared to the pre-pandemic period. Eight of 14 countries reported a drop in face-to-face consultations, while the remaining reported no change in the proportion of face-to-face consultations compared with the pre-pandemic period. A prominent reduction in face-to-face consultations was reported in Japan (95\%), the Philippines (95\%), Australia $(80 \%)$, and Indonesia $(50 \%)$. As an alternative, seven of
14 countries adopted video/telephonic consultations during the pandemic, with the highest adoption rate in the Philippines (95\%), Australia (80\%), Myanmar (70\%), and Indonesia (50\%).

\section{Discussion}

The COVID-19 pandemic significantly impacted the care of patients with HCC in healthcare institutions across the Asia-Pacific in general, but the extent of the impact varies and, to some extent, dependent on country-specific measures instituted to manage the pandemic. This study is the first of its kind to measure the extent of the impact of COVID-19 on the diagnosis and treatment of HCC patients in the Asia-Pacific with data obtained from 27 institutions in 14 countries. The number of countries that responded to the survey at the height of the initial wave of the pandemic in the Asia-Pacific was remarkable and contributed to the generalizability of the study findings. This study provides very useful data now as the region currently faces second and subsequent waves of infection in the pandemic.

During the period under study, the governments of these 14 countries instituted various levels of restrictions within their boundaries, of which travel restrictions (local, regional, international) are one of the most significant. In the initial phase of the pandemic, the number of patients with new diagnoses of HCC declined by $27 \%$ from the reference period before the pandemic. In only two 


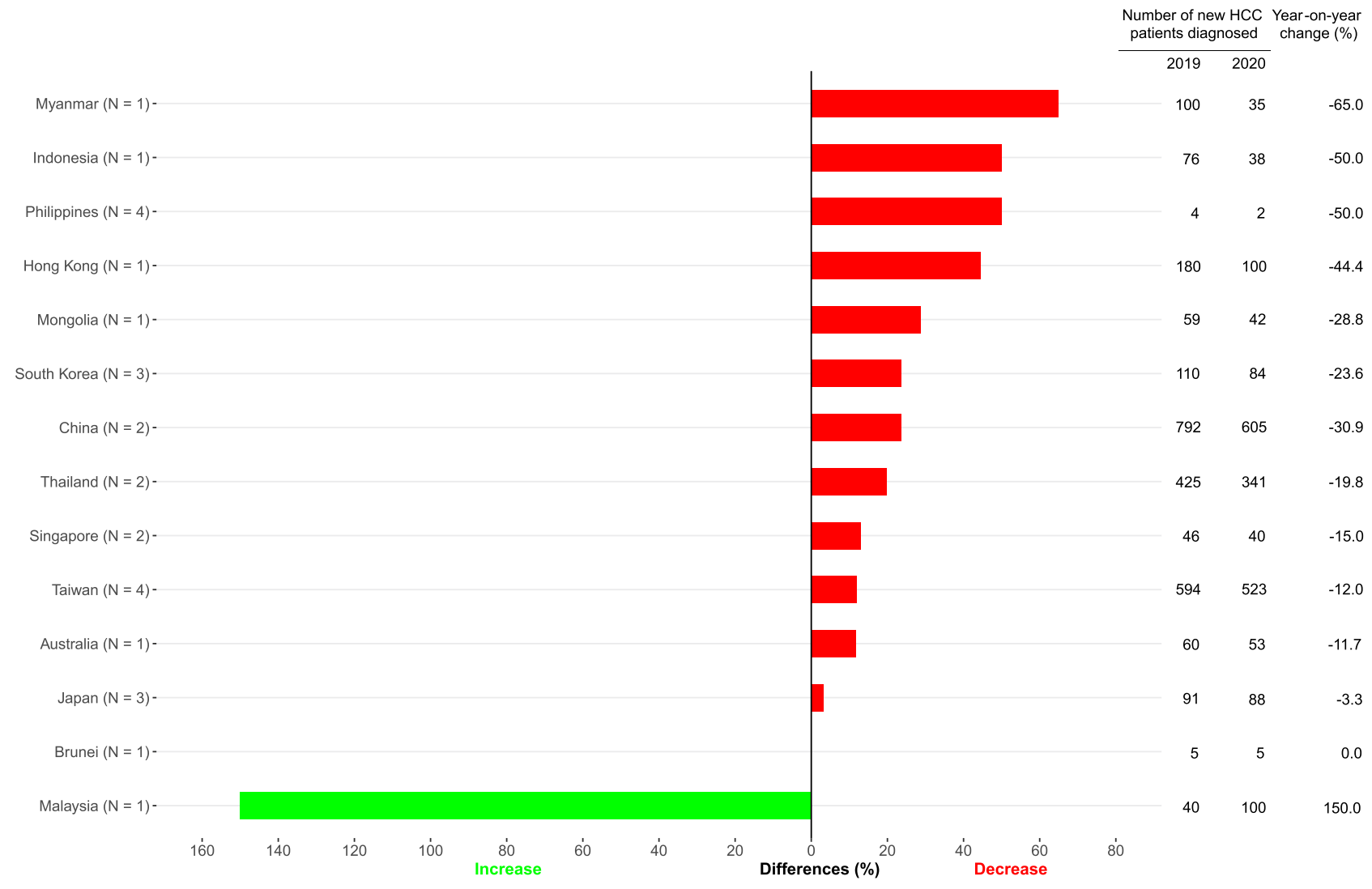

Figure I Comparing year-on-year change of new diagnosis of patients with hepatocellular carcinoma during the period of February to May in 2019 and 2020. Note: $\mathrm{N}$ represents the number of institutions that responded to the survey.

countries where there was no decrease in newly diagnosed $\mathrm{HCC}$ cases. The number of $\mathrm{HCC}$ cases diagnosed remained the same as the previous year only in Brunei, while Malaysia is the only country that showed an increase in the HCC cases. All other countries included in the study showed a decline.

To address the risk of disease transmission and difficulties with travel restrictions, the mode of consultation changed significantly in some countries. These changes leveraged technology that enabled patients to connect with doctors online. This study showed that remote consultations (video or phone consultation) and perceived difficulties in travel significantly reduced face-to-face consultations by $27 \%$. On the other hand, there was an $18 \%$ absolute increase in video or telephonic consultation from the pre-pandemic reference period. In line with the slowdown in the number of patients newly diagnosed with HCC, the study also sees no growth in patient enrollment in clinical trials in $\mathrm{HCC}$ during the pandemic compared to its previous year.

Some possible reasons for the negative impact that COVID-19 has on the diagnosis and treatment of HCC patients may be discerned from the survey. During the COVID-19 pandemic, social distancing and home confinement are two restrictions instituted by a number of governments, and these are largely complied with. Some countries have implemented complete lockdowns to control the spread of the disease. Patients with presumptive diagnoses of cancer may have been unable to visit hospitals for medical consultations, or their appointments may have been de-prioritized. In addition, general cancer screening programs were also suspended in various countries due to insufficient medical resources. ${ }^{17}$ Specifically, the shortage of ventilators and the high risks of infections during surgeries resulted in the postponement of nonemergency cancer surgeries and various other treatment procedures. $^{18}$

The results of this study are consistent with findings outside of the Asia-Pacific. A multicountry survey ${ }^{11}$ focusing on the impact of COVID-19 on HCC management in non-Asian countries found that $87 \%$ of centers modified their clinical practices during the pandemic $41 \%$ of centers modified diagnostic procedures, $81 \%$ deferred screening programs, and $50 \%$ canceled curative 
Table 2 Number of Newly Diagnosed Patients with Hepatocellular Carcinoma by Barcelona Clinic Liver Cancer (BCLC) Stages During the Period of February to May 2019 and February to May 2020

\begin{tabular}{|c|c|c|c|c|}
\hline Country (Number of Institutions) & BCLC Stage & Pre-Pandemic Period $^{\mathrm{a}} \mathrm{n}$ (\%) & Pandemic Period n (\%) & $\begin{array}{l}\text { Percentage } \\
\text { Change }^{b}\end{array}$ \\
\hline \multirow[t]{3}{*}{ All countries } & O/A & $853(34.1)$ & $751(37.2)$ & -12.0 \\
\hline & B & $824(33.0)$ & $638(31.6)$ & -22.6 \\
\hline & C & $821(32.9)$ & $631(3 \mid .2)$ & -23.1 \\
\hline \multirow[t]{3}{*}{ Australia $(\mathrm{N}=\mathrm{I})$} & O/A & $17(34.0)$ & $15(3 \mid .3)$ & -11.8 \\
\hline & B & $22(44.0)$ & $18(37.5)$ & -18.2 \\
\hline & C & II (22.0) & $15(31.3)$ & 36.4 \\
\hline \multirow[t]{3}{*}{ Brunei $(N=1)$} & O/A & $2(40.0)$ & $2(40.0)$ & 0.0 \\
\hline & B & $2(40.0)$ & $2(40.0)$ & 0.0 \\
\hline & $\mathrm{C}$ & I (20.0) & I (20.0) & 0.0 \\
\hline \multirow[t]{3}{*}{ China $(N=2)$} & O/A & $284(37.2)$ & $238(40.1)$ & -16.2 \\
\hline & B & $14 \mid(18.5)$ & $106(17.8)$ & -24.8 \\
\hline & $\mathrm{C}$ & $338(44.3)$ & $250(42.1)$ & -26.0 \\
\hline \multirow[t]{3}{*}{ Hong Kong $(\mathrm{N}=1)$} & O/A & $36(23.5)$ & $20(23.5)$ & -44.4 \\
\hline & B & $63(4 I .2)$ & $35(4 I .2)$ & -44.4 \\
\hline & C & $54(35.3)$ & $30(35.3)$ & -44.4 \\
\hline \multirow[t]{3}{*}{ Indonesia $(\mathrm{N}=\mathrm{I})$} & O/A & $2(33.3)$ & I (33.3) & -50.0 \\
\hline & B & $2(33.3)$ & I (33.3) & -50.0 \\
\hline & C & $2(33.3)$ & I (33.3) & -50.0 \\
\hline \multirow[t]{3}{*}{ Japan $(\mathrm{N}=3)$} & O/A & $56(60.9)$ & $54(61.4)$ & -3.6 \\
\hline & B & $18(19.6)$ & $17(19.3)$ & -5.6 \\
\hline & C & $18(19.6)$ & $17(19.3)$ & -5.6 \\
\hline \multirow[t]{3}{*}{ Malaysia $(\mathrm{N}=\mathrm{I})$} & O/A & $17(42.5)$ & $62(62.0)$ & 264.7 \\
\hline & B & $10(25.0)$ & $10(10.0)$ & 0.0 \\
\hline & C & $13(32.5)$ & $28(28.0)$ & 115.4 \\
\hline \multirow[t]{3}{*}{ Mongolia $(\mathrm{N}=\mathrm{I})$} & O/A & $8(13.6)$ & $6(14.3)$ & -25.0 \\
\hline & B & $20(33.9)$ & $14(33.3)$ & -30.0 \\
\hline & C & $31(52.5)$ & $22(52.4)$ & -29.0 \\
\hline \multirow[t]{3}{*}{ Myanmar $(\mathrm{N}=\mathrm{I})$} & O/A & $30(30.0)$ & $10(28.6)$ & -66.7 \\
\hline & B & $40(40.0)$ & $15(42.9)$ & -62.5 \\
\hline & C & $30(30.0)$ & $10(28.6)$ & -66.7 \\
\hline \multirow[t]{3}{*}{ Philippines $(\mathrm{N}=4)$} & O/A & $31(37.8)$ & 7 (25.9) & -77.4 \\
\hline & B & 27 (32.9) & II (40.7) & -59.3 \\
\hline & C & $24(29.3)$ & $9(33.3)$ & -62.5 \\
\hline \multirow[t]{3}{*}{ Singapore $(N=2)$} & O/A & $8(18.2)$ & $14(30.4)$ & 75.0 \\
\hline & B & $6(13.6)$ & $17(37.0)$ & 183.3 \\
\hline & C & $30(68.2)$ & $15(32.6)$ & -50.0 \\
\hline \multirow[t]{3}{*}{ South Korea $(\mathrm{N}=3)$} & O/A & $32(38.1)$ & $28(35.0)$ & -12.5 \\
\hline & B & $31(36.9)$ & $28(35.0)$ & -9.7 \\
\hline & C & $21(25.0)$ & $24(30.0)$ & 14.3 \\
\hline
\end{tabular}

(Continued) 
Table 2 (Continued).

\begin{tabular}{|l|l|l|l|l|}
\hline Country (Number of Institutions) & BCLC Stage & Pre-Pandemic Period ${ }^{\mathbf{a}} \mathbf{n}(\%)$ & Pandemic Period $\mathbf{n}$ (\%) $^{\text {Percentage }}$ \\
Change $^{\mathbf{b}}$
\end{tabular}

Notes: ${ }^{\text {Missing }} \mathrm{n}$ are imputed based on the percentage change in the new HCC cases during the pandemic compared to the same period during the prepandemic. ${ }^{b}($ Pandemic $n-$ Pre-pandemic $n) /$ Pre-pandemic $n \times 100$.

and/or palliative treatments. There was also a report of a surge in telephonic consultations in the majority of the centers. Similarly, an Italy-based study ${ }^{10}$ also found HCC treatment delay in $26 \%$ of the patients. For other types of cancer, various country-based studies reported delays in the diagnosis and treatments. For instance, research carried out in the Netherlands showed that the weekly incidence of cancer cases in The Netherlands Cancer Registry is decreased by up to $27 \%$ during the COVID19 outbreak compared with the period before the outbreak. ${ }^{7}$ Another report focusing on six common types of cancer in the United States reported a $46 \%$ decline in the weekly diagnosis of these cancers during the pandemic compared to the year before it. ${ }^{19}$ Similarly, following the institution of restrictions such as lockdowns and social distancing to control the spread of COVID-19, the referrals for suspected cancer patients were found to be decreased by $75 \%$ in the United Kingdom. $^{20}$ Our study found a significant reduction in elective surgery that would have consumed valuable hospital resources, including ICU beds and increased contact time with healthcare workers. There was however an increase in the use of oral systemic therapy for patients with HCC during the pandemic especially in BCLC B which decreases the use of hospital facilities and decreased patient contact time. These show a trend in adaptation in the management of HCC treatment towards less invasive outpatient treatments, which avoids long hospital stay as reported by an Italian study. ${ }^{21}$

Our study has several limitations. The study was based on aggregate institution-level data (not patientlevel data), which did not allow us to understand whether there were any systemic differences in patient profile at diagnosis during the pandemic versus those who did not. The study did not collect any follow-up data to understand any potentially negative impact on patient outcomes due to the COVID-19 pandemic. Furthermore, the study has not collected quantitative information on the extent of delay in terms of duration for HCC diagnosis and treatment initiation during the pandemic. This may vary from institution to institution. Also, the absolute percentage increase in treatment complication rate and reduction in the clinical trial enrollment rate may vary depending on the institution, and this information was not collected in the study. Another limitation was a small number of institutions from some countries or a small number of patients from the institution, which might not fully represent the situation in the whole country. An unexpected increase in HCC diagnosis during the pandemic in Malaysia could have resulted from this limitation or may have reflected a surge in $\mathrm{HCC}$ cases at a specialized cancer institution when other general hospitals have prioritized resources for COVID19. Furthermore, within the larger countries, there could be regional differences in COVID-19 prevention measures, and we were not able to evaluate the impact of such differences on the management of HCC patients in the current study. Therefore, such country-level data should be interpreted cautiously.

Many strategies were adopted in the initial phase of the pandemic (when this study was conducted) with short-term aims in the expectation that the pandemic would be swiftly brought under control. Data from our survey showed that the impact of these strategies on the clinical management of patients with $\mathrm{HCC}$ has generally been negative. Long-term sustainable strategies are required for a pandemic that may be long drawn out. 


\section{Conclusion}

The detailed results presented in this study on the impact of COVID-19 on the existing management of patients with HCC in the Asia-Pacific provide important data for healthcare authorities and healthcare service providers to re-assess the impact of policies on the diagnosis and treatment of HCC in line with the latest applicable management guidelines. ${ }^{22,23}$ This is particularly important as the region and the world now contend with subsequent waves of COVID-19 infections, and there is now a general expectation that it will take a while for the pandemic to run its course. Alternatives to major surgical therapy such as less invasive surgery or oral systemic treatment for HCC if appropriate and options to face-to-face consultations such as telephonic and video consultations will have to be increasingly adopted to address the urgent clinical needs uncovered by this study. Earlier assumptions that strict measures to control the pandemic are temporary in nature are no longer tenable, and there have to be longer-term and better thought-out plans to meet the urgent needs of patients with cancer during the pandemic.

\section{Data Sharing Statement}

The data is available upon reasonable request to the corresponding author.

\section{Ethics Approval and Informed Consent}

The paper is exempted from ethical committee approval.

\section{Acknowledgments}

The authors would like to acknowledge the following investigators and institutions for sharing their data. A/Prof Edmund Tse, Royal Adelaide Hospital, Australia. A/Prof Simone Strasser, Royal Prince Alfred Hospital, Australia. A/Prof Kenneth Kok Yuh Yen, Jerudong Park Medical Centre, Brunei. Prof Li Lequn, Guangxi Medical University Cancer Center, China. Prof Jiangtao Li, Zhejiang University School of Medicine, China. Prof Thomas Yau, Queen Mary Hospital, Hong Kong. Prof Tjakra Wibawa Manuaba, Sanglah General Hospital, Indonesia. Dr Ketut Mariadi, Sanglah General Hospital, Indonesia. Prof L.A. Lesmana, Medistra Hospital, Indonesia. Dr Cosmas Rinaldi A. Lesmana, Medistra Hospital, Indonesia. Prof Masatoshi Kudo, Kindai University Hospital, Japan. Prof Kiyoshi Hasegawa, The University of Tokyo, Japan. Dr Nobuyuki Takemura, National Center of Global Health and Medicine, Japan. Datuk Dr Harjit Singh, Hospital Selayang, Malaysia. Dr Suryati Mokhtar, Hospital Selayang,
Malaysia. Dr Ariunaa Khasbazar, National Cancer Centre of Mongolia, Mongolia. Prof Khin Maung Win, Yangon GI \& Liver Centre, Myanmar. Dr Rolley Rey Lobo, Davao Doctors' Hospital, Philippines. Dr Catherine Teh, Makati Medical Center, Philippines. Dr Ian Homer Y. Cua, St. Luke's Medical Center, Philippines. Dr Janus Ong, The Medical City, Philippines. Dr Vanessa De Villa, The Medical City, Philippines. Prof Kenneth Mak, Khoo Teck Puat Hospital, Singapore. Dr Tan Ming Yuan, Khoo Teck Puat Hospital, Singapore. Dr Toh Han Chong, National Cancer Centre Singapore, Singapore. Prof Sung-Bum Cho, Korea University Anam Hospital, South Korea. Prof Ho-Seong Han, Seoul National University Bundang Hospital, South Korea. Prof Jin-Mo Yang, St Vincent's Hospital, Catholic University Medical College, South Korea. Dr Peng ChengYuan, China Medical University Hospital, Taiwan. Dr PoChin Liang, National Taiwan University Hospital, Taiwan. Dr Chien-Hung Chen, National Taiwan University Hospital, Taiwan. Dr Rheun-Chuan Lee, Taipei Veterans General Hospital, Taiwan. Dr Yee Chao, Taipei Veterans General Hospital, Taiwan. Dr Pin-Nan Cheng, National Cheng Kung University Hospital, Taiwan. Dr Chanisa Chotipanich, Chulabhorn Hospital, Thailand. Dr Rawisak Chanwat, National Cancer Institute, Thailand.

\section{Author Contributions}

PKHC and MG conceived and designed the study. MG, CHC, LJH, MK, RC, SS, ZX, SHL, and PKHC designed the survey questionnaire. WHL and SHL acquired the data. WHL and MG analyzed and interpreted the data. All authors contributed to the drafting and revising of the article, have agreed on the journal to which the article will be submitted and approved the final manuscript for publication. All authors agree to be accountable for all aspects of the work in ensuring that questions related to the accuracy or integrity of any part of the work are appropriately investigated.

\section{Funding}

The study was funded by Duke-NUS, the "Estate of Tan Sri Khoo Teck Puat", SingHealth Duke-NUS Global Health Institute Research Grant, Duke-NUS Medical School (Duke-NUS/SDGHI_RGA(Khoo)/2020/0005) and NCCS Cancer Fund.

\section{Disclosure}

The authors report no conflicts of interest in this work. Masatoshi Kudo reports personal fees from Eli Lilly, Bayer, MSD, Bristol-Myers Squibb, Roche; grants and 
personal fees from Eisai, EA Pharma, Ono, Chugai; and grants from Gilead Sciences, Taiho, Sumitomo Dainippon Pharma, Takeda, Otsuka, AbbVie, outside the submitted work. Simone I Strasser reports personal fees from Eisai, AstraZeneca, Roche Pharmaceuticals, Bayer Healthcare, Guerbet, Ipsen, MSD, AbbVie, and Gilead Sciences, outside the submitted work.

\section{References}

1. World Health Organization. Cancer. Geneva: World Health Organization; 2021. Available from: https://www.who.int/en/newsroom/fact-sheets/detail/cancer. Accessed April 10, 2021.

2. Velavan TP, Meyer CG. The COVID-19 epidemic. Trop Med Int Health. 2020;25(3):278. doi:10.1111/tmi.13383

3. Wu Z, McGoogan JM. Characteristics of and important lessons from the coronavirus disease 2019 (COVID-19) outbreak in China: summary of a report of 72314 cases from the Chinese center for disease control and prevention. JAMA. 2020;323(13):1239-1242. doi:10.1001/jama.2020.2648

4. Richards M, Anderson M, Carter P, Ebert BL, Mossialos E. The impact of the COVID-19 pandemic on cancer care. Nature Cancer. 2020;1(6):565-567. doi:10.1038/s43018-020-0074-y

5. Doherty GJ, Goksu M, de Paula BH. Rethinking cancer clinical trials for COVID-19 and beyond. Nature Cancer. 2020;1(6):568-572. doi:10.1038/s43018-020-0083-x

6. Al-Quteimat OM, Amer AM. The impact of the COVID-19 pandemic on cancer patients. Am J Clin Oncol. 2020;43(6):452-455. doi:10.1097/COC.0000000000000712

7. Dinmohamed AG, Visser O, Verhoeven RH, et al. Fewer cancer diagnoses during the COVID-19 epidemic in the Netherlands. Lancet Oncol. 2020;21(6):750-751. doi:10.1016/S1470-2045(20) 30265-5

8. Raymond E, Thieblemont C, Alran S, Faivre S. Impact of the COVID-19 outbreak on the management of patients with cancer. Target Oncol. 2020;15(3):249-259. doi:10.1007/s11523-020-00721-1

9. Chan SL, Kudo M. Impacts of COVID-19 on liver cancers: during and after the pandemic. Liver Cancer. 2020;9(5):491-502. doi:10.1159/000510765

10. Iavarone M, Sangiovanni A, Carrafiello G, Rossi G, Lampertico P. Management of hepatocellular carcinoma in the time of COVID-19. Ann Oncol. 2020;31(8):1084-1085. doi:10.1016/j. annonc.2020.04.007

11. Muñoz-Martínez S, Sapena V, Forner A, et al. Assessing the impact of COVID-19 on liver cancer management (CERO-19). JHEP Rep. 2021;3(3):100260. doi:10.1016/j.jhepr.2021.100260

12. Harris PA, Taylor R, Thielke R, Payne J, Gonzalez N, Conde JG. A metadata-driven methodology and workflow process for providing translational research informatics support. $J$ Biomed Inform. 2009;42(2):377-381. doi:10.1016/j.jbi.2008.08.010
13. Harris PA, Taylor R, Minor BL, et al. The REDCap consortium: building an international community of software platform partners. J Biomed Inform. 2019;95:103208. doi:10.1016/j.jbi.2019.103208

14. SCRI. Singapore clinical research institute. Available from: https:// www.scri.edu.sg/services/. Accessed April 16, 2021.

15. Llovet JM, Brú C, Bruix J. Prognosis of Hepatocellular Carcinoma: The BCLC Staging Classification. Thieme Medical Publishers, Inc.; 1999:329-338.

16. Cohen J. Statistical Power Analysis for the Behavioral Sciences. Academic press; 2013.

17. Cancino RS, Su Z, Mesa R, Tomlinson GE, Wang J. The impact of COVID-19 on cancer screening: challenges and opportunities. JMIR Cancer. 2020;6(2):e21697. doi:10.2196/21697

18. Bartlett DL, Howe JR, Chang G, et al. Management of cancer surgery cases during the COVID-19 pandemic: considerations. Ann Surg Oncol. 2020;27:1717-1720.

19. Kaufman HW, Chen Z, Niles J, Fesko Y. Changes in the number of US patients with newly identified cancer before and during the coronavirus disease 2019 (COVID-19) pandemic. JAMA Netw Open. 2020;3(8):e2017267-e2017267. doi:10.1001/jamanetwork open. 2020.17267

20. IJzerman M, Emery J. Is a delayed cancer diagnosis a consequence of COVID-19? Melbourne: The University of Melbourne; 2020. Available from: https://pursuit.unimelb.edu.au/articles/is-a-delayedcancer-diagnosis-a-consequence-of-covid-19. Accessed April 17, 2021.

21. Santambrogio R, Farina G, D'Alessandro V, Iacob G, Gemma M, Zappa MA. Guidelines adaptation to the COVID-19 outbreak for the management of hepatocellular carcinoma. J Laparoendosc Adv Surg Tech. 2021;31(3):266-272. doi:10.1089/lap.2020.0559

22. Bollipo S, Kapuria D, Rabiee A, et al. One world, one pandemic, many guidelines: management of liver diseases during COVID-19. Gut. 2020;69(8):1369-1372. doi:10.1136/gutjnl-2020-321553

23. Wong GL-H, Wong VW-S, Thompson A, et al. Management of patients with liver derangement during the COVID-19 pandemic: an Asia-Pacific position statement. Lancet Gastroenterol Hepatol. 2020;5(8):776-787. doi:10.1016/S2468-1253(20)30190-4

24. Wikipedia. Travel restrictions related to the COVID-19 pandemic [updated August 24, 2021]. Available from: https://en.wikipedia. org/wiki/Travel_restrictions_related_to_the_COVID-19_pandemic. Accessed April 17, 2021.

25. Wikipedia. COVID-19 lockdowns [updated September 1, 2021]. Available from: https://en.wikipedia.org/wiki/COVID-19_lockdowns. Accessed April 17, 2021.

26. Wikipedia. Social distancing measures related to the COVID-19 pandemic [updated August 28, 2021]. Available from: https://en.wiki pedia.org/wiki/Social_distancing_measures_related_to_the_COVID19 pandemic. Accessed April 17, 2021.

27. Data OWi. Coronavirus pandemic (COVID-19) - the data [updated September 2, 2021]. Available from: https://ourworldindata.org/coro navirus-data. Accessed April 17, 2021.

\section{Publish your work in this journal}

The Journal of Hepatocellular Carcinoma is an international, peerreviewed, open access journal that offers a platform for the dissemination and study of clinical, translational and basic research findings in this rapidly developing field. Development in areas including, but not limited to, epidemiology, vaccination, hepatitis therapy, pathology and molecular tumor classification and prognostication are all considered for publication. The manuscript management system is completely online and includes a very quick and fair peer-review system, which is all easy to use. Visit http://www.dovepress.com/ testimonials.php to read real quotes from published authors. 